\title{
Palmitoylation is a post-translational modification of Alix regulating the membrane organization of exosome-like small extracellular vesicles
}

\author{
Daniele P. Romancino ${ }^{\mathrm{a}, 1}$, Valentina Buffa ${ }^{\mathrm{a}, 1}$, Stefano Caruso ${ }^{\mathrm{b}}$, Ines Ferrara ${ }^{\mathrm{a}}$, Samuele Raccosta ${ }^{\mathrm{c}}$, \\ Antonietta Notaro $^{\mathrm{a}}$, Yvan Campos ${ }^{\mathrm{d}}$, Rosina Noto ${ }^{\mathrm{c}}$, Vincenzo Martorana ${ }^{\mathrm{c}}$, Antonio Cupane ${ }^{\mathrm{e}}$, \\ Agata Giallongo $^{\mathrm{a}}$, Alessandra d'Azzo ${ }^{\mathrm{d}}$, Mauro Manno ${ }^{\mathrm{c}}$, Antonella Bongiovanni ${ }^{\mathrm{a}, *}$ \\ ${ }^{\mathrm{a}}$ Institute of Biomedicine and Molecular Immunology (IBIM), National Research Council (CNR) of Italy, Palermo, Italy \\ ${ }^{\mathrm{b}}$ UMR-1162, Functional Genomics of Solid Tumors, Inserm, Paris 1162, France \\ ${ }^{\mathrm{c}}$ Institute of Biophysics (IBF), National Research Council (CNR) of Italy, Palermo, Italy \\ d Department of Genetics, St. Jude Children's Research Hospital, Memphis, TN, USA \\ e Department of Physics and Chemistry, University of Palermo, Italy
}

\section{A R T I C L E I N F O}

\section{Keywords:}

extracellular vesicles (EVs)

Exosome

Alix (also known as PDCD6IP)

Skeletal muscle cells

Tetraspanin

S-palmitoylation

\begin{abstract}
A B S T R A C T
Background: Virtually all cell types have the capacity to secrete nanometer-sized extracellular vesicles, which have emerged in recent years as potent signal transducers and cell-cell communicators. The multifunctional protein Alix is a bona fide exosomal regulator and skeletal muscle cells can release Alix-positive nano-sized extracellular vesicles, offering a new paradigm for understanding how myofibers communicate within skeletal muscle and with other organs. S-palmitoylation is a reversible lipid post-translational modification, involved in different biological processes, such as the trafficking of membrane proteins, achievement of stable protein conformations, and stabilization of protein interactions.

Methods: Here, we have used an integrated biochemical-biophysical approach to determine whether S-palmitoylation contributes to the regulation of extracellular vesicle production in skeletal muscle cells.

Results: We ascertained that Alix is S-palmitoylated and that this post-translational modification influences its protein-protein interaction with CD9, a member of the tetraspanin protein family. Furthermore, we showed that the structural organization of the lipid bilayer of the small (nano-sized) extracellular vesicle membrane with altered palmitoylation is qualitatively different compared to mock control vesicles.

Conclusions: We propose that S-palmitoylation regulates the function of Alix in facilitating the interactions among extracellular vesicle-specific regulators and maintains the proper structural organization of exosome-like extracellular vesicle membranes.

General Significance: Beyond its biological relevance, our study also provides the means for a comprehensive structural characterization of EVs.
\end{abstract}

\section{Introduction}

Extracellular vesicles (EVs) are a heterogeneous population of membranous particles which have emerged in recent years as potent signal transducers and cell-cell communicators thanks to their content of proteins, lipids, and genetic material [1-3]. Based on the current knowledge of their biogenesis, EVs can be broadly divided into two main categories, exosomes and microvesicles, both involving membrane-trafficking processes. Until now, research on EVs has focused primarily on their role in the immune system and cancer. Recently, we

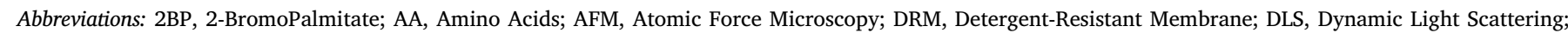

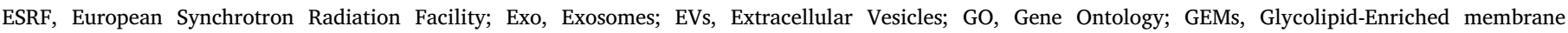

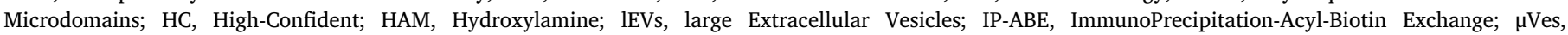

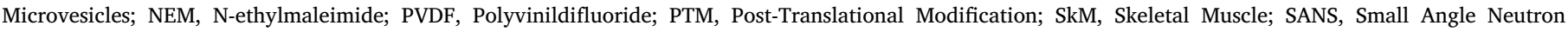
Scattering; SAXS, Small Angle X-ray Scattering; sEVs, small Extracellular Vesicles; TEMs, Tetraspanin-Enriched Microdomains

* Corresponding author at: Institute of Biomedicine and Molecular Immunology (IBIM), National Research Council (CNR) of Italy, via Ugo La Malfa, 153, Palermo, Italy.

E-mail address: bongiovanni@ibim.cnr.it (A. Bongiovanni).

${ }^{1}$ Equal contributors. 
have shown that skeletal muscle (SkM) cells can release Alix-positive exosome-like small EVs (sEVs), which suggested a function for these EVs in SkM biology [4]. We now want to decipher how muscle cells generate these nano-vesicles and how this process is regulated.

Alix (also known as Programmed cell death 6-interacting protein, PDCD6IP) is an evolutionarily conserved, ubiquitously expressed, multifunctional adaptor protein [5-7]. By binding selectively to a variety of protein partners (e.g., components of the endosomal sorting complexes required for transport, ESCRT, actin and cortactin) and a lipid (i.e., Bis(Monacylglycero)Phosphate, BMP, also inappropriately named LBPA for LysoBisPhosphatidic Acid), Alix has been implicated in many cellular processes, from endocytosis to cytoskeleton and membrane remodeling, and EV biogenesis [6,8-12]. The protein structure of Alix revealed a boomerang shape with a convex face (BRO1) encompassing a positively charged surface that might function as a membrane bending domain [13]. Alix functions in cellular events, such as cytokinesis, filopodia formation, membrane repair, viral and exosomal budding, which require the formation of negative membrane curvature and 100-nm scale membranous protrusions [8,14-16].

The subcellular architecture of SkM differs greatly from that of mononucleated cells. It is therefore predictable that preserving muscle structure and function requires a muscle-specific adaptation of known membrane trafficking and remodeling pathways [17,18]. For this reason, we and others have proposed that exosome biogenesis may differ according to the producing cell type and can also be sustained by direct exosome-like EVs budding from the plasma membrane, as it is the case of skeletal muscle and T cells $[1,4,19]$. Accordingly, highly ordered plasma membrane regions, such as lipid raft, tetraspanin-enriched microdomains (TEMs) and glycolipid-enriched membrane microdomains (GEMs), have been implicated in exosome biogenesis [20-22]. However, the plasma membrane domain(s) responsible for exosome-like sEV formation and structure are not known, nor are the coupled regulatory mechanisms that control membrane remodeling.

Tetraspanins, a transmembrane protein family highly enriched in exosomal membranes, can form homodimers and heterodimers, and can interact with cholesterol, gangliosides, as well as transmembrane and cytosolic signaling proteins $[23,24]$. They are organized in clusters on the membrane, generating TEMs that have the capacity to facilitate vesicular fusion or fission [25]. It has been reported by Booth and coworkers that, in lymphoblast and $\mathrm{T}$ cell lines, plasma membrane domains enriched in tetraspanin (e.g., CD81, CD63) and endosomal lipids (e.g., LBPA), serve as platforms for exosome budding [19]. The same group also demonstrated that S-palmitoylation and myristoylation tags target a highly oligomeric, recombinant cytoplasmic protein to secreted vesicles [26].

S-palmitoylation is a lipid post-translational modification (PTM), characterized by the attachment of a saturated fatty acid, palmitic acid (16:0), to specific cysteine residues of proteins [27]. Palmitoylation is distinguished from other lipid PTMs, such as prenylation and myristoylation, by its reversible nature. This PTM increases the hydrophobicity of proteins and regulates many key biological processes, such as the localization, conformation and stability of proteins at membranes, and protein-protein interaction. In the case of tetraspanins (e.g., CD9), S-palmitoylation is required for their interactions and functions [27-29]. However, the extent of the biological roles of this PTM remains to be fully elucidated.

Here, we have investigated whether S-palmitoylation regulates the membrane remodeling proteins involved in $\mathrm{EV}$ biogenesis in the context of TEMs, and the organization of the EV membrane. We applied an integrated biochemical-biophysical approach to determine that S-palmitoylation modulates the function of Alix and the integrity of SkMderived nano-sized SEV membranes.

\section{Materials and methods}

\subsection{Comparative analysis of publicly available datasets}

Data comparison and visualization were performed using R software (R Foundation for Statistical Computing, Vienna, Austria. https://www. R-project.org) with Bioconductor packages. UniProt accession numbers were assigned to the exosomal human protein dataset from ExoCarta in order to compare it with the S-palmitoylated protein dataset from SwissPalm. The intersection of the two datasets identified the exosomal proteins that are predicted to be S-palmitoylated. Fisher's exact test was used to confirm the different amounts of S-palmitoylation in the human and exosome proteomes. Gene ontology (GO) was used to annotate the $\mathrm{S}$-palmitoylated proteins for cellular component.

\subsection{Reagents and antibodies}

Hydroxylamine solution (HAM), N-ethylmaleimide (NEM), BrijO20, and 2-bromohexadecanoic acid (2BP), were purchased from SigmaAldrich (St. Louis, MO, USA). EZ-LinkTM BMCC-Biotin, Dynabeads protein G, Pierce streptavidin HRP conjugated and goat anti-rabbit Alexa Fluor 680 were purchased from Thermo Fisher Scientific (Rockford, IL, USA). Commercial antibodies included mouse anti-Alix for immunoblotting (clone 49/AIP1, BD Biosciences, San Jose, CA, USA) and anti-Alix for immunoprecipitations (clones 3A9 and 2H12, Santa Cruz Biotechnology, Santa Cruz, CA, USA); anti-CD63, clone H193 and anti-CD9, clone KMC8.8 (Santa Cruz Biotechnology); antiHsp70/Hsc70 (Novus Biologicals, Littleton, CO, USA); anti-Bax (SigmaAldrich); anti-PARP (Cell Signaling Technology, Leiden, The Netherlands); anti-pan Actin, clone AC-40 (Sigma-Aldrich).

\subsection{Extraction of proteins from $\mathrm{C} 2 \mathrm{C} 12$ cells and Alix co- immunoprecipitation}

The mouse C2C12 myoblast cells (ATCC-LGC, Wesel, Germany. ATCC $^{\oplus}$ Number: CRL-1772 ${ }^{\mathrm{TM}}$ ) [30] were plated at a density of $10^{6}$ myoblasts in $10 \mathrm{~cm}$ dish, and the next day these cells (approx. $2.510^{6}$ myoblasts $/ 10 \mathrm{~cm}$ dish) were induced to differentiation with $2 \%$ horse serum in place of $15 \%$ FCS. After 2 days of differentiation, cells were cultured for one day with or without $20 \mu \mathrm{M}$ 2BP. Cytoplasmic, membrane/organelle, nuclear, and cytoskeletal fractions were prepared using a ProteoExtract subcellular proteome extraction kit (Calbiochem, San Diego, CA, USA) according to the manufacturer's instructions, and EVs were separated as described in sub-section 2.4 below.

For IP-ABE analysis, cells and EVs were lysed using lysis buffer (0.5\% Triton X100, $50 \mathrm{mM}$ Tris-HCl, $\mathrm{pH} 7.5,5 \mathrm{mM} \mathrm{NaCl}, 2 \mathrm{mM} \mathrm{CaCl} 2$, $3 \mathrm{mM} \mathrm{KCl}, 1 \mathrm{mM} \mathrm{MgCl} 2,100 \mathrm{mM}$ sucrose), supplemented with complete EDTA-free Protease Inhibitor Cocktail (Roche, Mannheim, Germany), Phosphatase Inhibitor Cocktails 2 and 3 (Sigma-Aldrich), and freshly prepared $50 \mathrm{mM} \mathrm{N}$-Ethylmaleimide (NEM). The cell lysate was passed 8-9 times through a $26^{1 / 2}$ gauge syringe, gently shaken for $30 \mathrm{~min}$ at $4{ }^{\circ} \mathrm{C}$, and then centrifuged at $16000 \mathrm{~g}$ for $30 \mathrm{~min}$ at $4{ }^{\circ} \mathrm{C}$ in order to remove insoluble material. Clear supernatant was collected, and protein concentration was measured using the Pierce BCA protein assay kit (Thermo Fisher Scientific). IPs were performed using either $1 \mathrm{mg}$ of cell lysates (Fig. 2A, B) or $0.1 \mathrm{mg}$ of cell, $\mathrm{lEV}$ or sEV lysates (Fig. 2C, D).

For immunoprecipitation of the CD9-Alix complex, C2C12 cells were lysed with hypotonic buffer, cellular debris and nuclei were removed, and supernatants were centrifuged at $100,000 \mathrm{~g}$ for $1 \mathrm{~h}$ at $4^{\circ} \mathrm{C}$. Membrane pellets were resuspended in Brij (Sigma-Aldrich, Old tradename Brij $^{\mathrm{TM}} 98 \mathrm{~V}$ ) buffer (1\% BrijO20, $50 \mathrm{mM}$ Tris-HCl, pH 7.5, $150 \mathrm{mM} \mathrm{NaCl}, 2 \mathrm{mM} \mathrm{CaCl} 2$, and $1 \mathrm{mM} \mathrm{MgCl} 2$ ), incubated by rocking for $30 \mathrm{~min}$ at $4^{\circ} \mathrm{C}$, and centrifuged at $10,000 \mathrm{~g}$ for $10 \mathrm{~min}$ at $4^{\circ} \mathrm{C}$. Supernatants were quantified by BCA protein assay. In all cases the volumes of lysate samples were corrected to have same protein 
concentration $(2 \mathrm{mg} / \mathrm{ml})$. Protein extracts were precleared by adding $30 \mu \mathrm{l}$ of Dynabeads protein $\mathrm{G}$ to $1 \mathrm{mg}$ of proteins and incubating under agitation for $1 \mathrm{~h}$ at room temperature. Precleared supernatant was added to $3 \mu \mathrm{g}$ of monoclonal antibodies (anti-Alix, clones 3A9 and $2 \mathrm{H} 12$ ) and incubated by rocking overnight at $4{ }^{\circ} \mathrm{C}$. Subsequently, $30 \mu \mathrm{l}$ of Dynabeads protein $\mathrm{G}$ were added to each sample and to the negative control (CTRL, precleared supernatant with the isotype matching unrelated antibody anti-Flag), left under agitation for $2 \mathrm{~h}$ at room temperature, and washed five times with ice-cold Brij buffer. Afterwards, $2 \times$ SDS-PAGE non-reducing (125 mM Tris-HCl, pH 6.8, 4\% SDS, $20 \%$ glycerol, and $0.004 \%$ bromophenol blue) or reducing $(+5 \mathrm{mM}$ DTT) sample buffer were added to samples, boiled and loaded on 8-16\% sodium dodecyl sulfate-polyacrylamide gel electrophoresis (PAGESDS).

\subsection{EV separation}

EVs were separated and quantified according to a previously published method [4]. This concentration method included a penultimate centrifugation step in Eppendorf polypropylene conical tubes $(10,000 \mathrm{~g}$ for $30 \mathrm{~min}$ at $4{ }^{\circ} \mathrm{C}$, in Eppendorf rotor F-34-6-38) that allowed the removal/isolation of lEVs. Subsequently, nano-sized sEVs, comprised mainly of exosomes, were pelleted in Beckman Coulter polypropylene open top tubes $\left(118,000 \mathrm{~g}\right.$ for $70 \mathrm{~min}$ at $4{ }^{\circ} \mathrm{C}$, in Beckman rotor SW28). After washing, 10,000 or $118,000 \mathrm{~g}$ pellets were resuspended either in RIPA buffer or in PBS, for further immunoblotting or biophysics analyses, respectively. To estimate the amount of secreted vesicles, we quantified and compared the total protein content of the vesicle lysates using the BCA assay. Equal amounts of sEV lysates were loaded on SDSPAGE in reducing (+2-Mercaptoethanol) and non-reducing conditions to evaluate the homodimer forms of CD9 [31].

\subsection{Acyl-biotin exchange (ABE) onto PVDF membrane}

Immunoprecipitations were performed as described in sub-section 2.3 , with modifications. Briefly, precleared samples were incubated with anti-Alix (clone 3A9), and the immunoprecipitated proteins were washed five times with wash buffer containing $0.1 \%$ IGEPAL $^{\circledR}$ CA-630, $10 \mathrm{mM}$ Tris- $\mathrm{HCl}$, pH 7.5, $2 \mathrm{mM} \mathrm{CaCl} 2,3 \mathrm{mM} \mathrm{KCl}, 1 \mathrm{mM} \mathrm{MgCl} 2$, previously kept on ice. After the last wash, $2 \times$ SDS-PAGE reducing sample buffer were added to the beads; the beads were then heated for $5 \mathrm{~min}$ at $75-80^{\circ} \mathrm{C}$. Samples were separated from magnetic beads, and loaded on an $8-16 \%$ PAGE-SDS and electrophoresed at constant voltage (100 V), using Tris-Glycine SDS running buffer ( $25 \mathrm{mM}$ Tris-HCl, $192 \mathrm{mM}$ glycine, $0.1 \%$ SDS).

A modified ABE assay [32] was performed on immunoprecipitated proteins immobilized on PVDF membranes. Briefly, each sample was split into two parts before loading onto gels, one (1/3 of volume) was excluded from HAM treatment, and one ( $2 / 3$ of volume) was treated with HAM. This measure was taken to normalize for protein degradation caused by HAM treatment. After electrophoresis, separated proteins were transferred onto $0.2 \mu \mathrm{m}$ PVDF membranes (Amersham ${ }^{\mathrm{TM}}$ Hybond $^{\mathrm{TM}}$, GE Healthcare Life Science, Waukesha, WI, USA). The membranes were stained with Ponceau S (Sigma-Aldrich), washed with buffer A (50 mM HEPES, pH 7.4, $150 \mathrm{mM} \mathrm{NaCl,} \mathrm{0.5 \%} \mathrm{IGEPAL}{ }^{\circledR}$ CA630 ), and then incubated in buffer A supplemented with freshly prepared 20-50 mM NEM for $1 \mathrm{~h}$. Subsequently, the membranes were cut and treated separately with HAM buffer (1 M HAM, $50 \mathrm{mM}$ HEPES, pH 7.4, $150 \mathrm{mM} \mathrm{NaCl}, 0.5 \%$ IGEPAL $^{\circledast}$ CA-630) (+ HAM samples) or with buffer A (-HAM samples) for $1 \mathrm{~h}$. After this step, all the membranes were washed 3 times with buffer B (50 mM HEPES, pH 6.2, $150 \mathrm{mM}$ $\mathrm{NaCl}, 0.5 \%$ IGEPAL $^{\oplus}$ CA-630) supplemented with $1 \mathrm{mM}$ EDTA for $10 \mathrm{~min}$, then membranes were incubated with Biotin buffer $(0.3 \mu \mathrm{M}$ BMCC-biotin in buffer B) for $1 \mathrm{~h}$. Lastly, membranes were washed with PBS. A blocking buffer containing 3\% BSA in TBS with $0.05 \%$ Tween-20 (TBST) was used to block the PVDF membranes, and after 2 washes with TBST, the re-assembled membranes ( + or -HAM) were incubated with streptavidin HRP conjugated diluted 1:20000 in blocking buffer. Subsequently, the membranes were washed once with blocking buffer for $30 \mathrm{~min}$, and 3-4 times with TBST for $20 \mathrm{~min}$. In order to detect the originally palmitoylated protein bands, the membranes were incubated for $5 \mathrm{~min}$ using a chemiluminescent substrate (Pierce ECL western blotting substrate, Thermo Scientific), and were then exposed for 1-5 min to Amersham Hyperfilm ${ }^{\mathrm{TM}}$ ECL (GE Healthcare Life Science).

\subsection{Western blotting}

PVDF membranes were probed with primary antibodies, and after washing steps were incubated with Alexa Fluor 680 (Invitrogen, Life Technologies, Paisley, United Kingdom) anti-rabbit or IRDye 800 (LICOR Biosciences, Lincoln, USA) anti-mouse IgGs. To determine signal intensity from infrared bands we used an Odyssey Infrared Imaging System (LI-COR) and the LI-COR imaging software. Intensity of the Ponceau bands was quantified using ImageJ software and used to normalize signals from specific antibodies.

\subsection{Analysis of size distribution by light scattering}

Scattered light intensity and its time autocorrelation function $g_{2}(t)$ were measured simultaneously on different vesicle samples at the temperature $T=20^{\circ} \mathrm{C}$ by using a Brookhaven BI-9000 correlator (Brookhaven Instruments, Holtsville, NY, USA), equipped with a solidstate laser tuned at $\lambda_{o}=532 \mathrm{~nm}$. The samples were diluted to a final total protein content of 33 and $66 \mu \mathrm{g} / \mathrm{ml}$ for exosome-like sEVs and lEVs, respectively, in order to avoid vesicle interaction and multiple scattering artefacts. Apart from an instrumental parameter $\beta, g_{2}(t)$ directly yields the field correlation function $g_{1}(t)\left(g_{2}(t)=1+\beta\left|g_{1}(t)\right|^{2}\right)$, which is analyzed to determine the weight averaged distribution $a_{q}(\sigma)$ of hydrodynamic diameters $\sigma: g_{1}(t)=\int a_{q}(\sigma)$ exp. $\left\{-D(\sigma) q^{2} t\right\}$ d $\sigma$. In the latter expression, $q=4 \pi \tilde{n} \lambda_{o}{ }^{-1} \sin (\vartheta / 2)$ is the scattering vector, with $\vartheta=90^{\circ}$ being the scattering angle and $\tilde{n}=1.3367$ the medium refractive index, and $D(\sigma)$ is the diffusion coefficient, related to $\sigma$ by the Stokes-Einstein relation: $D(\sigma)=k_{B} T(3 \pi \eta \sigma)^{-1}$, where $k_{B}$ is the Boltzmann constant, $T$ is the temperature and $\eta$ is the solvent viscosity [33]. $a_{q}(\sigma)$ is here shaped as a simple two-parameter gamma function with average $\quad \sigma_{0}: \quad a_{q}(\sigma) \mathrm{d} \sigma=\Gamma(\alpha)^{-1} \alpha^{\alpha} D\left(\sigma_{0}\right)^{-\alpha} D(\sigma)^{\alpha-1} \exp \{-\alpha D(\sigma) D$ $\left.\left(\sigma_{0}\right)^{-1}\right\} \mathrm{dD}(\sigma)$. Absolute values for scattered intensity (Rayleigh ratio) were obtained by normalization with respect to toluene, whose Rayleigh ratio at $532 \mathrm{~nm}$ was taken as $28 \cdot 10^{-6} \mathrm{~cm}^{-1}$. Intensity absolute values were used to estimate the total content of small and large EVs.

Since light scattered intensity is proportional to the square of the mass of scatterers, the measured size distribution is dominated by larger mass objects. This intrinsic peculiarity of light scattering technique needs to be taken into account when reading the number averages, notwithstanding the robustness of DLS to identify the complete size distribution. Therefore, while the maximum of the distribution indicates the mass weighted most abundant species, the most numerous species are certainly included in the range at left of the distribution maximum.

\subsection{Atomic force microscopy (AFM) images}

Vesicle samples were diluted to a total protein content of $1 \mu \mathrm{g} / \mathrm{ml}$ in PBS and a $30 \mu \mathrm{l}$ drop was deposited on a freshly cleaved mica substrate for $10 \mathrm{~min}$ at room temperature. Vesicle images were immediately acquired by using an Asylum Research Cypher Microscope (Oxford Instruments, Abingdon, United Kingdom) operating in liquid tapping mode (resolution $512 \times 512$, scan rate $0.5 \mathrm{~Hz}$ ). We used Olympus BLAC40TS cantilevers with a typical tip curvature radius of $10 \mathrm{~nm}$ (nominal spring constant $0.1 \mathrm{~N} / \mathrm{m}$, resonance frequency $25 \mathrm{kHz}$ in liquid). The free oscillation amplitude was set at $10 \mathrm{~nm}$ and images were acquired at $50 \%$ larger of the free amplitude. AFM experiments were 
performed at the PSCM facility of the European Synchrotron Radiation Facility (Grenoble, France).

\subsection{Small angle $X$-ray scattering (SAXS) and small angle neutron scattering (SANS) measurements}

Small Angle X-ray and Neutron Scattering (SAXS and SANS) are complementary to microscopy techniques, since they provide a wealth of structural information displayed in the reciprocal space or as a function of the momentum transfer q. Hence, the length scales associated to the whole vesicle size and shape are probed at low q, while those related to the bilayer organization are displayed at high $\mathrm{q}$ (around and above $\mathrm{q}=1 \mathrm{~nm}^{-1}$ ).

SAXS data were collected at different synchrotron facilities: beamline SWING of Synchrotron Soleil, Gif-sur-Yvette, France; beamline SAXS of synchrotron Elettra, Trieste, Italy; beamline BM29 of European Synchrotron Radiation Facility (ESRF), Grenoble, France. In all cases, small and large EV samples in PBS from different preparations were frozen at $-20^{\circ} \mathrm{C}$. The day before the SAXS/SANS experiments, samples were thawed, pooled, and suspended in PBS at total protein content between 0.5 and $1.0 \mathrm{mg} / \mathrm{ml}$. Samples were injected in a $1 \mathrm{~mm}$ optical path capillary kept at $25^{\circ} \mathrm{C}$ by using the respective injection methods (automatic autosampler at ESRF, automatic injection with air bubble separating different volume of the sample at Soleil; static manual loading at Elettra). Several (seven to ten) consecutive measurements of $60 \mathrm{~s}$ were performed for each sample by moving the sample under the beam. At Elettra, where the sample could not be moved each single acquisition lasted $10 \mathrm{~s}$. Since successive exposures showed the same scattering pattern, indicating no radiation damage, the data were averaged over the different exposures. Since vesicle solutions are isotropic samples, 2D detector images were radially integrated to obtain the intensity scattering pattern I(q) as a function of the magnitude of the momentum transfer, or scattering vector, $q=(4 \pi \sin \theta) / \lambda$, where $2 \theta$ is the scattering angle, and $\lambda$ is the wavelength of the incident X-ray beam. From each profile a background was subtracted by taking the intensity profile of the buffer, which was measured before and after each sample by using the same capillary.

SANS measurements provide conceptually the same kind of structural information than SAXS; indeed, the data are collected and processed in an analogous way, including 2D detector imaging, radial averaging and buffer subtraction. The main difference consists in the type of radiation/matter interaction: while X-ray interacts with atomic electron densities, neutrons interact with a smaller part contained in atom nuclei. Small Angle Neutron Scattering data were collected at beamline D16 of Institute Laue Langevin (ILL), Grenoble, France. The samples were resuspended in fully deuterated PBS, incubated for $48 \mathrm{~h}$ at $4{ }^{\circ} \mathrm{C}$, and loaded into $1 \mathrm{~mm}$ optical path quartz cuvette. Measurements were performed with an average duration of $20 \mathrm{~min}$ by using different sample detector distance, covering different ranges of scattering vector q.

\subsection{Statistical analysis}

Data were expressed as mean \pm S.D. and were evaluated using Student's $t$-test. Mean differences were considered statistically significant when $p$ values were $<0.05{ }^{*}$ ).

\section{Results and discussion}

\subsection{S-palmitoylated proteins are enriched in the exosome subcellular compartment}

To evaluate the extent to which S-palmitoylation is functionally linked to exosome biogenesis, we first conducted a comparative analysis of publicly available data. We used the SwissPalm manually curated protein S-palmitoylation database (http://swisspalm.epfl.ch),

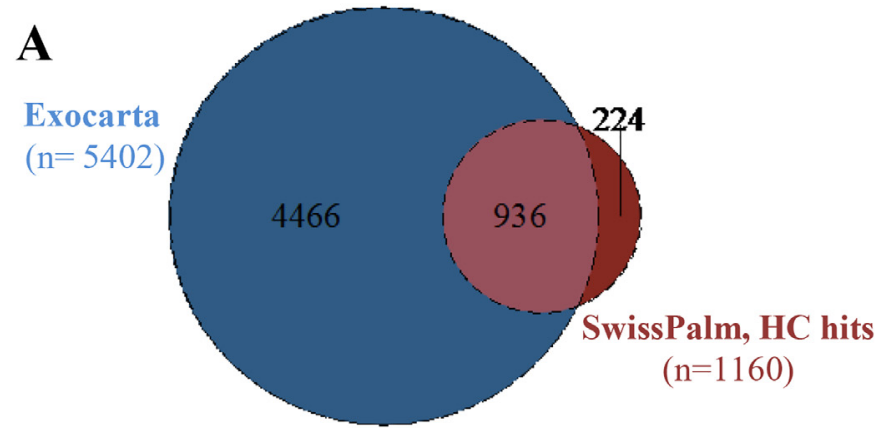

\section{B PALMITOYLATED NON-PALMITOYLATED}

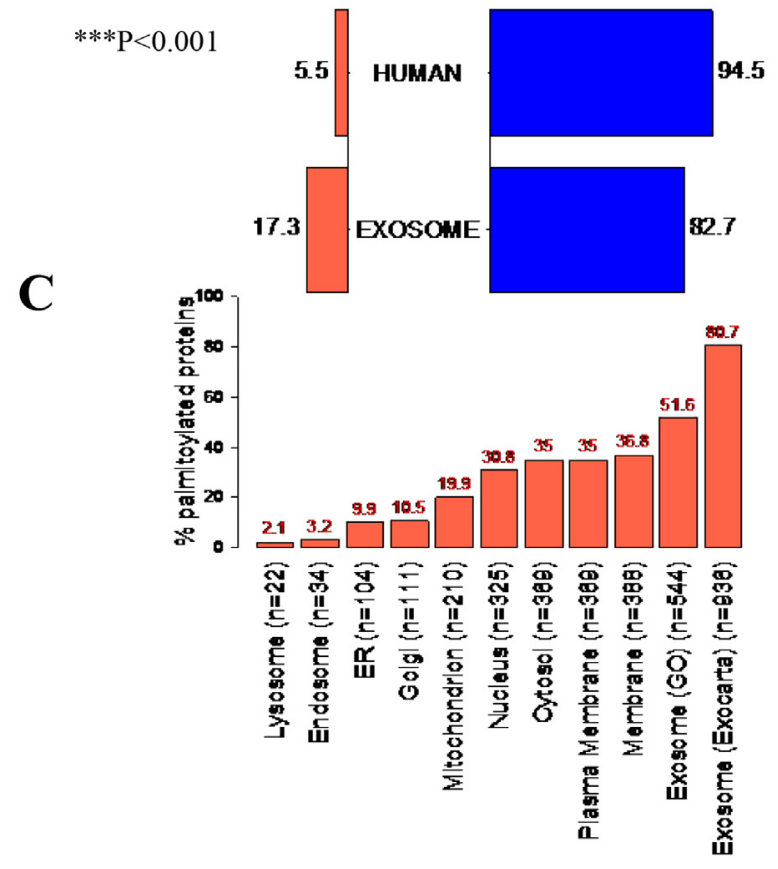

Fig. 1. Bioinformatic analyses of S-palmitoylation in exosomes.

(A) Venn diagram representing the overlap between exosomal human proteins from ExoCarta and S-palmitoylated proteins with high confidence (HC) from SwissPalm. (B) Pyramid plot showing the different distribution of S-palmitoylation between the human proteome and exosomal protein content (Fisher's exact test) ( $p$ value $<0.001$ ). (C) Distribution of S-palmitoylation hits in the different cellular compartments.

which encompasses 1160 S-palmitoylated, high-confident (HC) hits from palmitoyl-proteome screens, and the ExoCarta exosome database (http://www.exocarta.org), which includes information on exosomal human proteins from diverse cell types and body fluids [34,35]. The overlap of these two datasets showed that a surprisingly high number of HC S-palmitoylated proteins are annotated in ExoCarta as exosomal human proteins (Fig. 1A). The list of S-palmitoylated exosomal proteins is provided in Supplementary Table S1.

Furthermore, although $10 \%$ of the human proteome undergoes Spalmitoylation [36], and only 5.5\% of the HC S-palmitoylated proteins are distributed in the human proteome, there is about a three-fold enrichment in the number of these proteins in the exosomal protein content ( $p$ value $<0.001$, Fisher's exact test) (Fig. 1B).

We then investigated the distribution of S-palmitoylated proteins across different cellular compartments using gene ontology (GO) annotation [37-39] for cellular components. Consistent with the previous findings, exosomes contained the highest percentage of S-palmitoylated proteins $(80.7 \%)$, compared to all the other cellular compartments (Fig. 1C). The high percentage of palmitoylated exosomal proteins suggests that this PTM is a distinctive signature of exosomal proteins, a 
finding in line with recent reports showing the role of a prenylation/ palmitoylation tag in targeting a recombinant, highly oligomeric cytoplasmic protein on exosomes [26], as well as the importance of palmitoylation for the sorting of the viral protein LMP1 in exosomes [40]. The enrichment of S-palmitoylated proteins in exosomes is similar to what occurs within the core complexes of caveolae and TEM microdomains [22]. TEM membrane platforms have been implicated in exosome biogenesis and virus assembly and are characterized by a liquid-ordered membrane structure, also induced by virtue of palmitoylation-mediated tetraspanin clustering [20-22].

\subsection{Endogenous Alix is palmitoylated in SkM cells}

Alix is a bona fide membrane remodeling protein prone to dimerizing and oligomerizing [10,41,42], and it is one of the most abundant components of exosome-like sEVs. Thus, we focused on this exosome key player to examine whether S-palmitoylation is an Alix's PTM. For this purpose, we first queried the palmitoyl-proteomic data reported in Swiss Palm for Alix S-palmitoylation and found four palmitoyl-proteome studies in which both human and mouse Alix were reported as Spalmitoylated proteins [43-46]. Although palmitoylation, unlike other types of lipidation, does not require a distinct sequence motif in the substrate, it has been proposed that a 51 amino acid (AA) protein fragment centered on a cysteine encompasses a latent motif that serves as a palmitoylation site [47]. By using a bioinformatics tool, SeqPalm [47], we identified the predicted palmitoylated cysteine residue (HuCys231); notably, Cys231 in human Alix is exposed on the protein surface in close proximity to the hydrophobic loop, which is important for membrane binding [6], and is surrounded by a 51 AA fragment highly conserved across vertebrates (Fig. S1).

Next, to investigate whether endogenous Alix is S-palmitoylated in differentiated SkM cells and SkM-derived EVs, we developed a modified immunoprecipitation-Acyl-Biotin Exchange (IP-ABE) method [32] on immunoprecipitated Alix, immobilized on polyvinildifluoride (PVDF) membranes. This assay uses hydroxylamine (HAM) to cleave palmitate from immunoprecipitated proteins, exchanging it with biotin and allowing for the detection of palmitoylated proteins by streptavidin. Because HAM treatment usually degrades part of the immunoprecipitated proteins, we performed it on immunoprecipitated Alix immobilized on PVDF membranes, rather than in solution, in order to minimize protein loss. We then compared the levels of palmitoylated-Alix among different samples by quantifying the palmitoylated-Alix band (detected by streptavidin-HRP) and the total Alix band (detected by anti-Alix antibody), and then by calculating the ratio of Palm-Alix/Alix. This modified, semi-quantitative assay allowed for the analysis of the endogenous palmitoylated-Alix in cell and EV lysates. As SkM cellular model, we used C2C12 myotubes at day 3 of differentiation, either untreated or treated with 2-bromopalmitate (2BP), an inhibitor of the acylation/deacylation protein machinery. $2 \mathrm{BP}$ is a non-metabolizable palmitate analog that blocks palmitate incorporation into proteins; different cell lines are usually incubated overnight with this inhibitor at a final concentration of $100 \mu \mathrm{M}$ [48]. Among different dose and timing conditions we applied a treatment with $20 \mu \mathrm{M} 2 \mathrm{BP}$ for $24 \mathrm{~h}$ that did not induce any overt morphological changes in the myotubes compared to untreated cells (Fig. S2A). Moreover, the myotubes remained viable, as demonstrated by the levels of the apoptotic markers BAX and PARP, which remained unchanged in the cytoplasmic and nuclear fractions of 2BP treated and untreated myotubes, respectively (Fig. S2B).

Following treatment with 2BP, myotubes were lysed and the endogenous Alix was immunoprecipitated using an anti-Alix monoclonal antibody. The modified IP-ABE assay showed that endogenous Alix was $S$-palmitoylated in myotubes, as evidenced by the streptavidin-positive bands in IP-ABE samples, which were absent in the control lanes where the essential ABE reagent hydroxylamine was omitted (Fig. 2A). Furthermore, altering palmitoylation with $2 \mathrm{BP}$ significantly reduced the palmitoylation level of Alix confirming that the endogenous protein carries this PTM (Fig. 2A, B).

\subsection{Alix in nano-sized sEVs is palmitoylated to a larger extent than cellular and IEV Alix}

We then investigated whether Alix was also palmitoylated within myotube-derived EVs. Large and small EVs were isolated from myotubes by sequential ultracentrifugation. Ultracentrifugation is the most common EV purification technique; it allows the removal/isolation of lEVs, which comprise microvesicles, for the subsequent pelleting of small, nano-sized EVs (sEVs), which include exosomes [49,50]. Consistently, we will use this definition of sEVs and lEVs in this paper.

Equal amounts of small EV-, large EV- and total cell-lysates were immunoprecipitated using anti-Alix antibody, and $\mathrm{ABE}$ assays were performed on the immunoprecipitated material. Comparing the ratio of palmitoylated Alix to the total Alix immunoprecipitated from the three lysates, we found that the levels of palmitoylated Alix were significantly higher in small than in large EVs or total cell-lysates (Fig. 2C, D). Thus, we can conclude that Alix in the exosome-like sEVs is palmitoylated to a larger extent than Alix species included in the large EVs or within cells.

\subsection{Alix interacts with $C D 9$, and $2 B P$ affects their binding}

It has been shown previously that several members of the tetraspanin protein family are S-palmitoylated and regulate protein sorting into exosomes [20]. We therefore wanted to assess the distribution of Alix and the tetraspanin family members, CD9 and CD63, in myotubederived sEVs after 2BP treatment.

Treatment with 2BP induced a significant reduction of CD9 homodimers [31] in exosome-like sEVs (Fig. 3A, B). Furthermore, under the same non-reducing condition, in place of the Alix band of $96 \mathrm{kDa}$, a distinct dimer-like Alix-related band of approximately $200 \mathrm{kDa}$ was observed (Fig. 3A, B). We also found a slight reduction of Alix and CD9 monomers after palmitoylation inhibition with 2BP, compared to untreated cells (Fig. 3A, B). In contrast, the CD63 levels increased, while HSP70 and actin remained unchanged (Fig. 3A, B). The combined observations that the largest fraction of palmitoylated Alix segregated with sEVs and that palmitoylation inhibition with $2 \mathrm{BP}$ drastically reduced the amount of a dimer-like Alix form in these sEVs, point to a key role of palmitoylation in driving Alix transfer to these nanovesicles, possibly by regulating Alix dimerization, as it does for tetraspanins [23]. Similarly to other tetraspanin family members, dimeric Alix represents the active form of the protein that interacts with protein partners (e.g., ESCRT-III CHMP4 polymers) and localizes to membranes; the Alix dimer functions as a scaffolding protein during membrane remodeling processes, and disruption of Alix dimerization inhibits the release of HIV [41,42]. Because of the similar behavior of Alix and tetraspanin and the role of TEMs in EV biogenesis, we asked whether Alix would take part in the tetraspanin web via interaction with CD9. Endogenous Alix was immunoprecipitated using two different anti-Alix monoclonal antibodies (clone 3A9 or 2H12) [51]. We found that the CD9 and Alix endogenous proteins co-precipitate, using both anti-Alix antibodies, from myotube membranes extracted with the Brij98 detergent (Fig. 3C, D), which preserves protein interactions within the TEMs [52]. This result identifies Alix as a novel interactor of CD9 in the TEMs. Furthermore, we found that the interaction between the two proteins depends on their palmitoylation status, because 2BP-altered palmitoylation resulted in a significant reduction of Alix-CD9 binding (Fig. 3C, D). Indeed, although similar amounts of Alix were immunoprecipitated from control and 2BP-treated myotube extracts, we detected a reduced amount of CD9 upon treatment with 2BP (Fig. 3C, D), indicating that S-palmitoylation supports the interaction among Alix and CD9, as it occurs for tetraspanin complexes in TEMs.

To date, the only PTMs known to regulate the structure and function of Alix are ubiquitination and phosphorylation [8,53-55]. Furthermore, our group demonstrated that the binding of OZZ, the substrate binding 

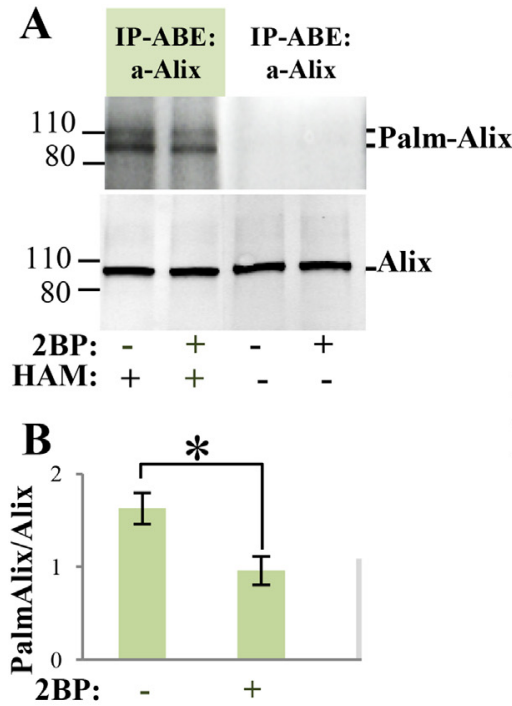

C
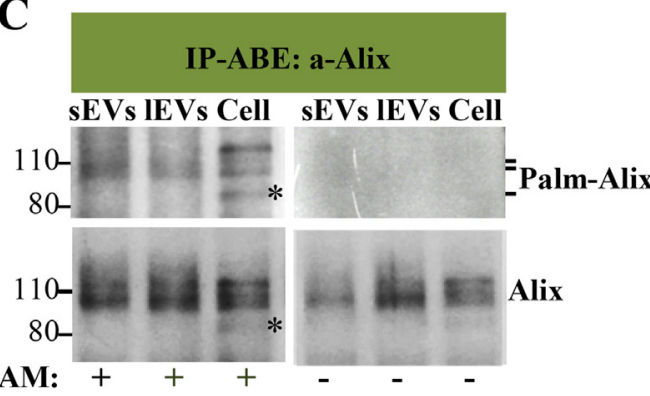

D

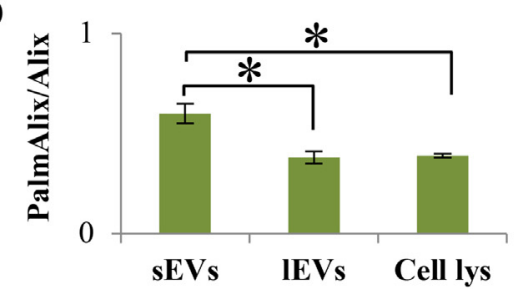

Fig. 2. Alix is S-palmitoylated in SkM cells, and the S-palmitoylation level of Alix in exosome-like sEVs is higher compared to that in lEVs or within cells.

(A) A representative IP-ABE assay showing S-palmitoylation of the endogenous Alix in cellular extracts of $\mathrm{C} 2 \mathrm{C} 12$ myotubes, omission of hydroxylamine (-HAM) is shown as a control. IPs were performed using $1 \mathrm{mg}$ of cell lysates. A decreased level of Alix palmitoylation is observed after 2BP treatment. (B) Quantification of S-palmitoylation by densitometric scanning of Palm-Alix (detected by streptavidinHRP) and Alix (detected by anti-Alix immunoblot) bands; the ratio of Palm-Alix/Alix was calculated in three independent experiments. (C) A representative blot of IP-ABE assay showing higher levels of palmitoylated Alix in myotube-derived exosome-like sEVs compared to lEVs and cellular (Cell) extracts, isolated from $\mathrm{C} 2 \mathrm{C} 12$ myotubes. IPs were performed using $0.1 \mathrm{mg}$ of lysates. Omission of HAM is shown as a control. The asterisk indicates the cleavage product of Alix. (D) Quantification by densitometric scanning of Palm-Alix (detected by streptavidin-HRP) and Alix

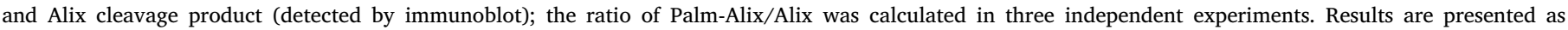
average $\pm \mathrm{SD},{ }^{*} p<0.05$.

component of the RING type ubiquitin ligase complex OZZ-E3, changes Alix's conformation and makes the protein accessible for further ubiquitination [8]. Our current results suggest that S-palmitoylation also regulates Alix interaction to CD9 and the localization of Alix in sEVs.

\subsection{The inhibition of palmitoylation affects EV number and size distribution}

Firstly, we used atomic force microscopy (AFM) to characterize myotube-derived sEVs in solution. Fig. 4A shows the typical image of a small, nanosized EV with an approximately spherical shape, an apparent width of $w^{\prime} \sim 200 \mathrm{~nm}$ and an apparent height of $h \sim 70 \mathrm{~nm}$. The actual width $w$ was estimated as $\sim 125 \mathrm{~nm}$ by applying a standard correction for a parabolic tip on a step $w=w^{\prime}-\left(8 R_{\text {tip }} h\right)^{0.5}$, where $\mathrm{R}_{\text {tip }} \sim 10 \mathrm{~nm}$ is the curvature radius of the AFM tip [56]. We may also argue that vesicles deposition on the substrate caused a squeezing on the vertical direction and a correspondent broadening on the planar axis. Indeed, if this is the case, the shape would have been moderately altered in all the deposited vesicles. For instance, by assuming that the overall volume is not altered, an oblate object on the substrate with main axes of 70 and $125 \mathrm{~nm}$, alike the vesicle in the Fig. 4A, would correspond to a spherical object in solution with a diameter of $100 \mathrm{~nm}$. These results are consistent with our previous electron microscopy measurements of fixed myotube-derived exosome-like small EVs isolated in the same conditions and with the same procedure [4].

Next, to examine the effects of blocking S-palmitoylation on the number and size distribution of EVs, we applied dynamic light scattering (DLS) analyses on myotube-derived small and large vesicles. The analyses of 2BP-treated and untreated myotube-derived sEVs by DLS showed an average hydrodynamic diameter of about $150 \mathrm{~nm}$ for 2BPtreated and -untreated sEV preparations, which is consistent with the
A

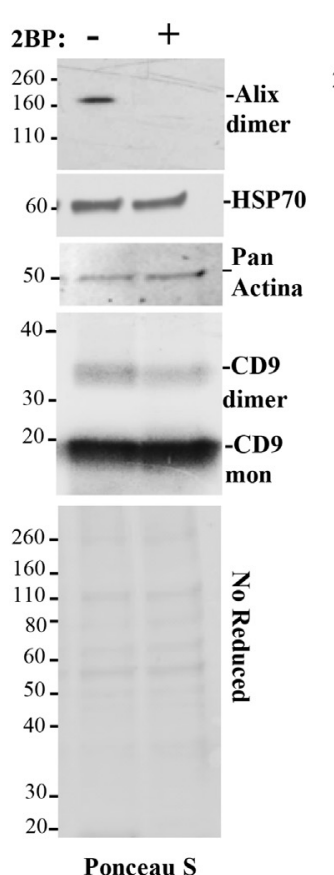

B

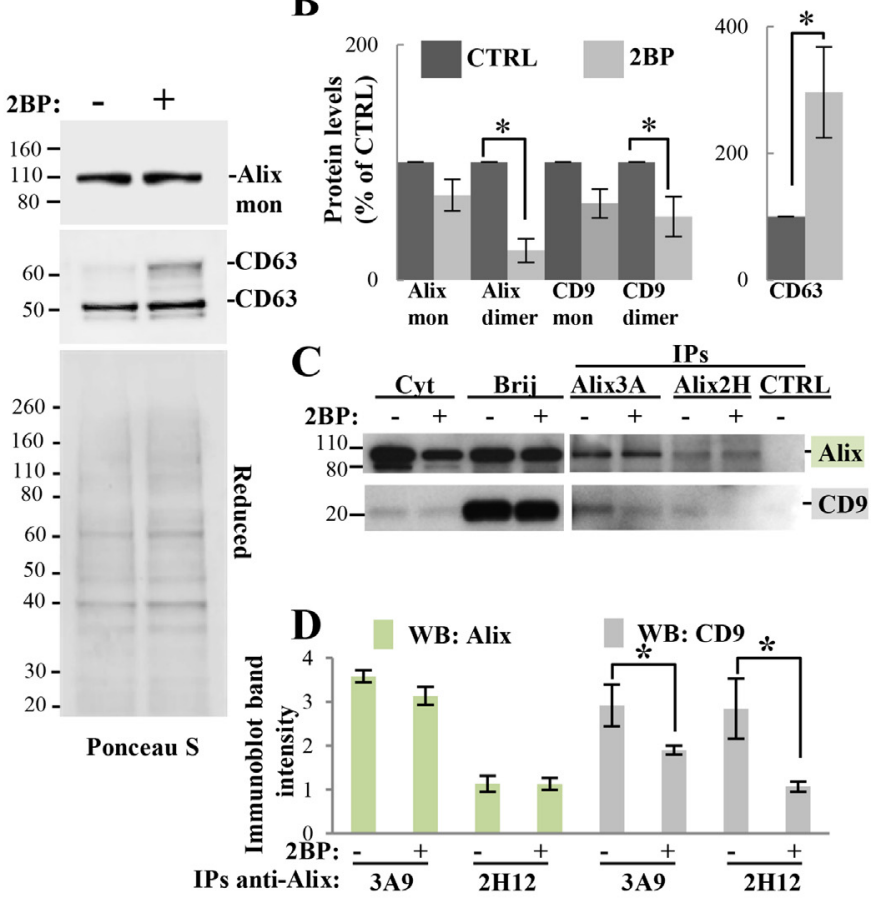

Fig. 3. S-palmitoylation influences cargo distribution of exosome-like sEVs and Alix-CD9 interaction. (A) Representative immunoblots of exosome-like sEV lysates in non-reducing and reducing conditions showing that $2 \mathrm{BP}$ treatment induced a significant reduction of Alix and CD9 dimer-like forms and an increase of CD63 levels. (B) Quantification of immunoblots by densitometric scanning; band signals were normalized to the protein levels determined by Ponceau S staining. (C) Representative immunoblots of cytosol (Cyt) and Brij98-soluble membranes (Brij), and the IPs of Brij extracts using anti-Alix antibodies (clones 3A9 and 2H12). Alix and CD9 interact, and the palmitoylation inhibition $(+2 \mathrm{BP})$ affects their binding. The isotype matching antibody anti-Flag (IgG1) was used as negative control (CTRL). (D) Quantification of Alix and CD9 bands in immunoblots of IPs. Results are presented as average \pm SD of three independent experiments, *p $<0.05$. 

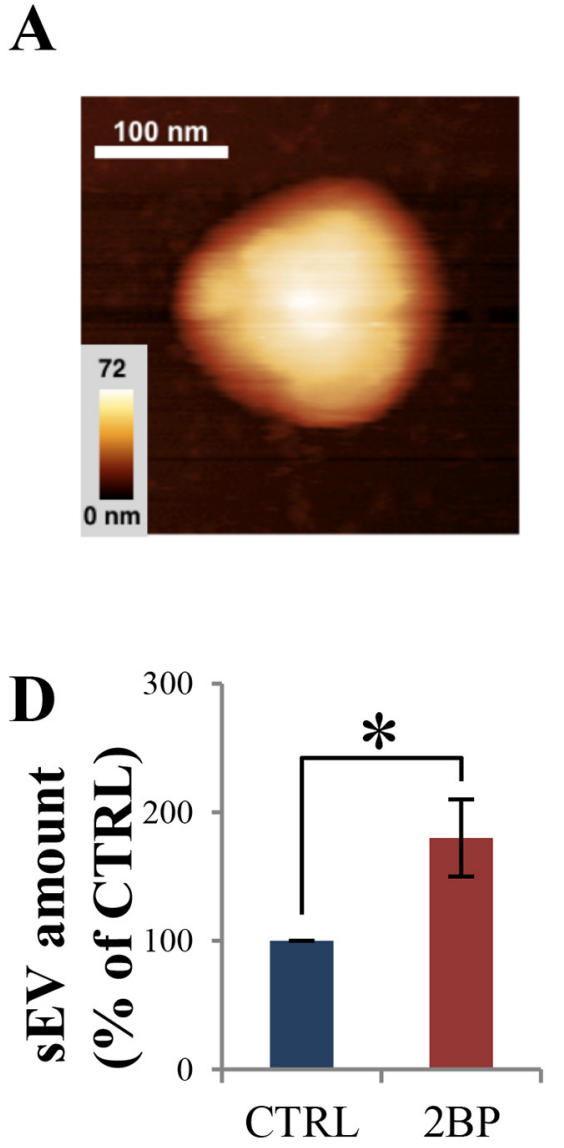
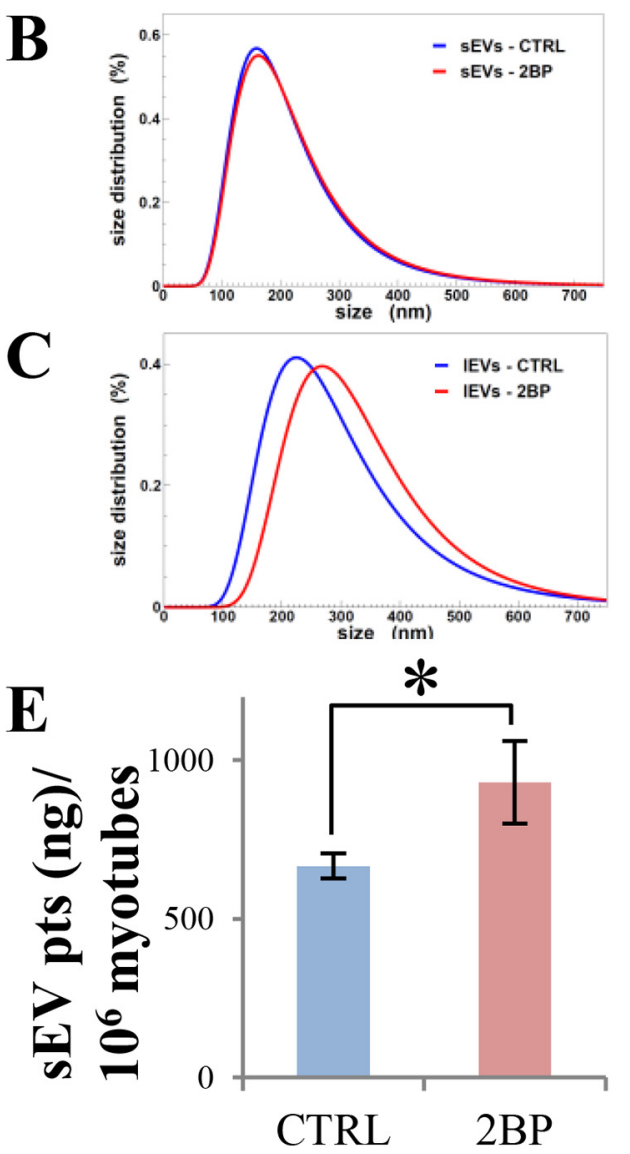

Fig. 4. Inhibition of S-palmitoylation influences the number and size distribution of sEVs.

(A) AFM image of a typical exosome-like sEVs. The solid white bar and the colored scale in the inset indicate the width and height of the pictured vesicle, respectively. (B) Typical size distributions measured by DLS of control (blue) and 2BP treated (red) sEVs, obtained from the same EV preparation. (C) Size distributions measured by DLS of control (blue) and 2BP treated (red) lEVs, from the same EV preparation of panel $\mathrm{B}$.

2BP treatments induce a significant increase of sEV number (D) and protein concentration (E). (D) The number of sEVs was determined by DLS. (E) The relative EV protein content was determined by BCA assay. Results are presented as average \pm SD of three independent experiments, ${ }^{*} \mathrm{p}<0.05$. size of exosomes (Fig. 4B). Similar distribution profiles have been observed in several sample preparations $(n=5)$. In addition, 2BP treatment generated a slight increase in the number of vesicles of larger size, included in the lEV preparations (Fig. 4C). DLS analyses also revealed a significant increase in the number of sEVs following 2BP treatment (Fig. 4D), data that were corroborated by an increase in the total protein concentration of 2BP-treated sEVs, compared to control (Fig. 4E). The number and total protein content of lEVs were not affected by 2BP treatment (data not shown). These observations indicate that the alteration of palmitoylation by $2 \mathrm{BP}$ mainly influences the $\mathrm{SEV}$ population, inducing a general large secretion of myotube-derived nano-sized sEVs and only a slight increased proportion of larger lEVs than mock control EVs. A similar deregulated sEV biogenesis has already been observed when exosome regulators are silenced [57] or when lipids, such as cholesterol, are depleted [58].

\subsection{Analysis of the organization of SkM-derived SEV membranes}

The enrichment of S-palmitoylated proteins in exosomes (Fig. 1) is similar to what occurs within the core complexes of caveolae and TEM microdomains [22]. TEM membrane platforms have been implicated in exosome biogenesis and virus assembly and are characterized by a liquid-ordered membrane structure, also induced by virtue of palmitoylation-mediated tetraspanin clustering [20-22]. The exosome membrane is not identical to its parent lipid bilayer, as evidenced by their unique rigid lipid bilayer membrane; it is selectively enriched in sphingolipids, cholesterol, glycerophospholipids, ceramide, tetraspanins, membrane proteins associated with membrane rafts and integrins [59]. Similarly, the exosome membrane has peculiar features as it is resistant to freeze-thaw cycles or to high saline concentration [60]. SAXS has already been used to describe the organization of the lipid bilayer of synaptic vesicles or vesicles of synthetic origin [61-63]. Thus, to evaluate the functional significance of the enrichment of S-palmitoylated proteins in exosomes with respect to the organization of the native SEV membrane, we performed synchrotron radiation SAXS experiments on exosome-like sEVs and lEVs derived from C2C12 myotube cultures. For this purpose, C2C12 myotube-derived EVs, either untreated or treated with $2 \mathrm{BP}$, were subjected to SAXS analyses (see Materials and Methods section for detail). Fig. 5 shows scattering profiles (the SAXS intensity function I(q) versus $\mathrm{q}$ ) for a typical 2BP-treated or not sEV or lEV sample. In all measurements, we observed a peculiar scattering profile with a hump at about $1.2 \mathrm{~nm}^{-1}$; above this value, the SAXS curve related to exosome-like sEVs exhibits a characteristic pattern, quite distinct from that of either lEVs or 2BP-treated sEVs (Fig. 5A). Intriguingly, the same structural feature was observed on different sample preparations and by using different beam lines, for a total of three replicates: SAXS at Elettra and SWING at Soleil (Fig. 5A), and BM29 at ESRF (Fig. 5B). This finding was remarkable, given that we were analyzing a complex natural system with no external control regarding the actual composition of vesicles.

As recalled in the Material and Method section, SAXS displays all the structural information in the reciprocal space of the momentum transfer $\mathrm{q}$; thus, the scattering profile around and above $\mathrm{q}=1.2 \mathrm{~nm}^{-1}$ is mainly generated by length scales around and below $2 \pi / \mathrm{q}=5.2 \mathrm{~nm}$, which may corresponds to a typical bilayer size decorated with protruding biomolecules. Notwithstanding this trivial consideration, we aimed to gain further and more robust information on the nature of this structural feature; thus, we performed combined SANS and SAXS experiments by using the same sample preparations, namely the myotubederived sEVs (Fig. 5B). To our knowledge, SANS studies have been previously performed using model membrane systems; the only two SANS analyses of biological membranes were performed on detergentresistant membrane (DRM) vesicles and Bacillus subtilis membranes $[64,65]$. Here, we performed SANS measurements of the intact 


\section{A}

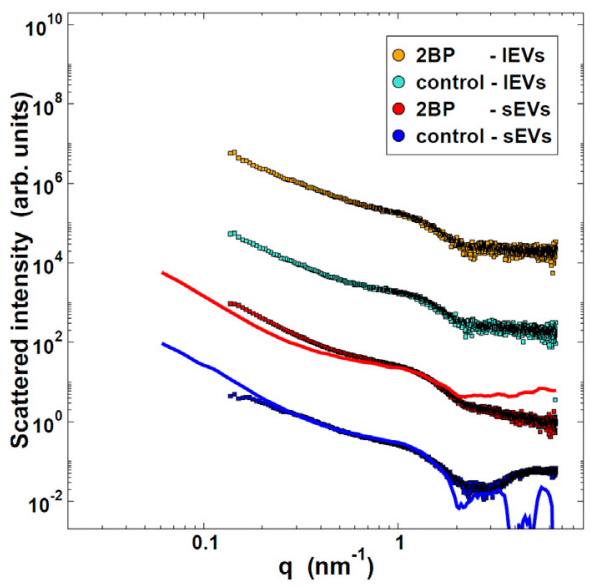

B

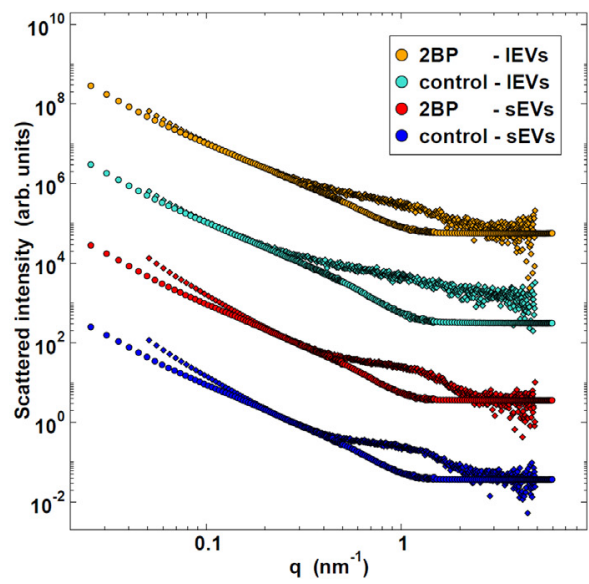

Fig. 5. Analysis of the membrane organization of EVs, and the influence of S-palmitoylation inhibition. EVs treated or not with 2BP were analyzed by SAXS and SANS; the patterns of different samples are color-coded as in the legends. Patterns are vertically shifted for clarity. (A) SAXS profiles performed at Elettra (squares) and Soleil (solid lines) showing a qualitatively different scattering pattern of control for exosome-like sEVs compared to lEVs and 2BPtreated exosome-like sEVs. (B) SAXS profiles performed at ESRF (diamonds) and SANS profiles performed at ILL (circles) of control myotube-derived exosome-like sEVs, lEVs treated or not with $2 \mathrm{BP}$. biological membrane of myotube-derived exosome-like sEVs. Our SANS results show that, in fully deuterated buffer, the hump typical of SAXS patterns disappeared (Fig. 5B). In our experimental condition, the SANS pattern does not highlight the contrast among the different vesicle components and the fully deuterated solvent. On the other hand, the electron density contrasts between vesicle components and the solvent are different among them (e.g., 12.1, 14.4, and 7.2 vs. $9.410^{-6} \AA^{-2}$ for proteins, phospholipid head-groups, and hydrophobic tails, respectively $v s$. aqueous solvent), and hence are detectable in SAXS measurements. In particular, the difference in the scattering length densities of the head-groups and hydrophobic tails of the lipid bilayer results in high contrast and detectability in SAXS measurements. Thus, we can confidently ascribe the hump typical of the SAXS patterns to the structure of the lipid bilayer, confirming what has been found in other systems [63]. It is worth noting that such unequivocal identification of SAXS pattern as due to the lipid bilayer becomes highly reliable, since both SAXS and SANS measurements are performed on the same preparation and within $48 \mathrm{~h}$ from resuspension, exploiting the physical proximity of the two facilities for SAXS and SANS (ESRF and ILL, respectively).

A more detailed analysis, in terms of typical onion shell models and SANS analyses with contrast variation, is ongoing but is beyond the scope of the present work. Here, we prefer to exploit the main qualitative features fostered by these data in a model-free approach. The SAXS data indicate a characteristic membrane organization of sEVs that is significantly different from that of $1 \mathrm{EVs}$ and 2BP-treated sEVs; we related these qualitative differences to changes in the organization of the lipid bilayer. This result corroborates and expands recent data, based on EV staining with a fluorescent, polarity-sensitive lipid probe, which showed that exosomal membranes are characterized by a high membrane lipid order compared to microvesicles [66]. Interestingly, high membrane lipid order is typically found in membrane nano- and micro-domains (e.g., TEMs, lipid rafts) at the immunological synapses and at sites of cell adhesion, viral entry, or viral budding. The distinct membrane organization of exosome-like sEVs compared to that of lEVs may represent a unique feature underlying the role of exosomes in intercellular signaling and/or biogenesis.

Taken together, our results provide insights into the role of S-palmitoylation in maintaining a proper membrane structure organization of exosome-like sEVs, in addition to regulating the proper localization of Alix in SkM sEVs and supporting the interactions among exosomespecific regulators/biomarkers (i.e., Alix and CD9).

Supplementary data to this article can be found online at https:// doi.org/10.1016/j.bbagen.2018.09.004.

\section{Author contributions}

$\mathrm{AB}, \mathrm{MM}$ conceived the research; $\mathrm{AB}, \mathrm{AC}, \mathrm{DR}, \mathrm{VM}, \mathrm{MM}, \mathrm{SR}$ designed the experiments; AB, DR, VB, SC, VM, MM, AG, Ad'A analyzed the data; $\mathrm{AB}, \mathrm{DR}, \mathrm{VB}, \mathrm{IF}, \mathrm{SR}, \mathrm{YC}, \mathrm{AN}, \mathrm{SC}, \mathrm{RN}, \mathrm{VM}, \mathrm{MM}$ performed the research; AB, Ad'A, AG wrote the paper; DR, MM, VM, YC, SC, SR contributed new reagents or analytic tools.

\section{Acknowledgements}

We thank Richard Burket for editing the manuscript, the kind support of Luca Costa and Fabio Comin (ESRF) and the PSCM platform at ESRF and ILL (Grenoble, France), and Mariana Valente (Pierre and Marie Curie University - Paris 6) for support in graphical abstract design. The research leading to these results has been funded by the Italian Ministry for Education, Universities, and Research in the framework of the Flagship Project NanoMAX.

\section{References}

[1] M. Simons, G. Raposo, Exosomes-vesicular carriers for intercellular communication, Curr. Opin. Cell Biol. 21 (2009) 575-581.

[2] M. Yanez-Mo, P.R. Siljander, Z. Andreu, A.B. Zavec, F.E. Borras, E.I. Buzas, K. Buzas, E. Casal, F. Cappello, J. Carvalho, E. Colas, A. Cordeiro-da Silva, S. Fais, J.M. Falcon-Perez, I.M. Ghobrial, B. Giebel, M. Gimona, M. Graner, I. Gursel, M. Gursel, N.H. Heegaard, A. Hendrix, P. Kierulf, K. Kokubun, M. Kosanovic, V. Kralj-Iglic, E.M. Kramer-Albers, S. Laitinen, C. Lasser, T. Lener, E. Ligeti, A. Line, G. Lipps, A. Llorente, J. Lotvall, M. Mancek-Keber, A. Marcilla, M. Mittelbrunn, I. Nazarenko, E.N. Nolte-'t Hoen, T.A. Nyman, L. O'Driscoll, M. Olivan, C. Oliveira, E. Pallinger, H.A. Del Portillo, J. Reventos, M. Rigau, E. Rohde, M. Sammar, F. Sanchez-Madrid, N. Santarem, K. Schallmoser, M.S. Ostenfeld, W. Stoorvogel, R. Stukelj, S.G. Van der Grein, M.H. Vasconcelos, M.H. Wauben, O. De Wever, Biological properties of extracellular vesicles and their physiological functions, J. Extracell. vesicles 4 (2015) 27066.

[3] J. Kowal, G. Arras, M. Colombo, M. Jouve, J.P. Morath, B. Primdal-Bengtson, F. Dingli, D. Loew, M. Tkach, C. Thery, Proteomic comparison defines novel markers to characterize heterogeneous populations of extracellular vesicle subtypes, Proc. Natl. Acad. Sci. U. S. A. 113 (2016) E968-E977.

[4] D.P. Romancino, G. Paterniti, Y. Campos, A.D. Luca, V.D. Felice, A. d'Azzo, A. Bongiovanni, Identification and characterization of the nano-sized vesicles released by muscle cells, FEBS Lett. 587 (2013) 1379-1384.

[5] G. Odorizzi, The multiple personalities of Alix, J. Cell Sci. 119 (2006) 3025-3032.

[6] H. Matsuo, J. Chevallier, N. Mayran, I. Le Blanc, C. Ferguson, J. Faure, N.S. Blanc, S. Matile, J. Dubochet, R. Sadoul, R.G. Parton, F. Vilbois, J. Gruenberg, Role of LBPA and Alix in multivesicular liposome formation and endosome organization, Science 303 (2004) 531-534.

[7] C. Bissig, J. Gruenberg, ALIX and the multivesicular endosome: ALIX in Wonderland, Trends Cell Biol. 24 (2014) 19-25.

[8] A. Bongiovanni, D.P. Romancino, Y. Campos, G. Paterniti, X. Qiu, S. Moshiach, V. Di Felice, N. Vergani, D. Ustek, A. d'Azzo, Alix protein is substrate of Ozz-E3 ligase and modulates actin remodeling in skeletal muscle, J. Biol. Chem. 287 (2012) 12159-12171.

[9] M.F. Baietti, Z. Zhang, E. Mortier, A. Melchior, G. Degeest, A. Geeraerts, Y. Ivarsson, F. Depoortere, C. Coomans, E. Vermeiren, P. Zimmermann, G. David, Syndecansyntenin-ALIX regulates the biogenesis of exosomes, Nat. Cell Biol. 14 (2012) $677-685$.

[10] R.D. Fisher, H.Y. Chung, Q. Zhai, H. Robinson, W.I. Sundquist, C.P. Hill, Structural and biochemical studies of ALIX/AIP1 and its role in retrovirus budding, Cell 128 (2007) 841-852. 
[11] Y. Campos, X. Qiu, E. Gomero, R. Wakefield, L. Horner, W. Brutkowski, Y.G. Han, D. Solecki, S. Frase, A. Bongiovanni, A. d'Azzo, Alix-mediated assembly of the actomyosin-tight junction polarity complex preserves epithelial polarity and epithelial barrier, Nat. Commun. 7 (2016) 11876.

[12] V. Mercier, M.H. Laporte, O. Destaing, B. Blot, C.M. Blouin, K. Pernet-Gallay, C. Chatellard, Y. Saoudi, C. Albiges-Rizo, C. Lamaze, S. Fraboulet, A. Petiot, R. Sadoul, ALG-2 interacting protein-X (Alix) is essential for clathrin-independent endocytosis and signaling, Sci. Rep. 6 (2016) 26986.

[13] J. Kim, S. Sitaraman, A. Hierro, B.M. Beach, G. Odorizzi, J.H. Hurley, Structural basis for endosomal targeting by the Bro1 domain, Dev. Cell 8 (2005) 937-947.

[14] E. Morita, L.A. Colf, M.A. Karren, V. Sandrin, C.K. Rodesch, W.I. Sundquist, Human ESCRT-III and VPS4 proteins are required for centrosome and spindle maintenance, Proc. Natl. Acad. Sci. U. S. A. 107 (2010) 12889-12894.

[15] Q. Zhai, M.B. Landesman, H. Robinson, W.I. Sundquist, C.P. Hill, Structure of the Brol domain protein BROX and functional analyses of the ALIX Brol domain in HIV-1 budding, PLoS One 6 (2011) e27466.

[16] A.J. Jimenez, P. Maiuri, J. Lafaurie-Janvore, S. Divoux, M. Piel, F. Perez, ESCRT machinery is required for plasma membrane repair, Science 343 (2014) 1247136.

[17] L.L. Scheffer, S.C. Sreetama, N. Sharma, S. Medikayala, K.J. Brown, A. Defour, J.K. Jaiswal, Mechanism of $\mathrm{Ca}(2)(+)$-triggered ESCRT assembly and regulation of cell membrane repair, Nat. Commun. 5 (2014) 5646.

[18] S. Charrin, M. Latil, S. Soave, A. Polesskaya, F. Chretien, C. Boucheix, E. Rubinstein, Normal muscle regeneration requires tight control of muscle cell fusion by tetraspanins CD9 and CD81, Nat. Commun. 4 (2013) 1674.

[19] A.M. Booth, Y. Fang, J.K. Fallon, J.M. Yang, J.E. Hildreth, S.J. Gould, Exosomes and HIV Gag bud from endosome-like domains of the T cell plasma membrane, J. Cell Biol. 172 (2006) 923-935.

[20] D. Perez-Hernandez, C. Gutierrez-Vazquez, I. Jorge, S. Lopez-Martin, A. Ursa, F. Sanchez-Madrid, J. Vazquez, M. Yanez-Mo, The intracellular interactome of tetraspanin-enriched microdomains reveals their function as sorting machineries toward exosomes, J. Biol. Chem. 288 (2013) 11649-11661.

[21] F. Thuma, S. Heiler, M. Schnolzer, M. Zoller, Palmitoylated claudin7 captured in glycolipid-enriched membrane microdomains promotes metastasis via associated transmembrane and cytosolic molecules, Oncotarget 7 (2016) 30659-30677.

[22] W. Yang, D. Di Vizio, M. Kirchner, H. Steen, M.R. Freeman, Proteome scale characterization of human S-acylated proteins in lipid raft-enriched and non-raft membranes, Mol. Cell. Proteomics 9 (2010) 54-70.

[23] S. Charrin, S. Jouannet, C. Boucheix, E. Rubinstein, Tetraspanins at a glance, J. Cell Sci. 127 (2014) 3641-3648.

[24] S.R.a.M. Zöller, The functional importance of tetraspanins in exosomes, in: H. G. Zhang (Ed.), Emerging Concepts of Tumor Exosome-Mediated, Cell-Cell Communication, 69 Springer Science + Business Media, NewYork, 2013, pp. 69-105.

[25] M.E. Hemler, Targeting of tetraspanin proteins-potential benefits and strategies, Nat. Rev. Drug Discov. 7 (2008) 747-758.

[26] B. Shen, N. Wu, J.M. Yang, S.J. Gould, Protein targeting to exosomes/microvesicles by plasma membrane anchors, J. Biol. Chem. 286 (2011) 14383-14395.

[27] S. Blaskovic, M. Blanc, F.G. van der Goot, What does S-palmitoylation do to membrane proteins? FEBS J. 280 (2013) 2766-2774.

[28] C. Salaun, J. Greaves, L.H. Chamberlain, The intracellular dynamic of protein palmitoylation, J. Cell Biol. 191 (2010) 1229-1238.

[29] I. Levental, D. Lingwood, M. Grzybek, U. Coskun, K. Simons, Palmitoylation regulates raft affinity for the majority of integral raft proteins, Proc. Natl. Acad. Sci. U. S. A. 107 (2010) 22050-22054.

[30] H.M. Blau, G.K. Pavlath, E.C. Hardeman, C.P. Chiu, L. Silberstein, S.G. Webster, S.C. Miller, C. Webster, Plasticity of the differentiated state, Science 230 (1985) $758-766$.

[31] O.V. Kovalenko, X. Yang, T.V. Kolesnikova, M.E. Hemler, Evidence for specific tetraspanin homodimers: inhibition of palmitoylation makes cysteine residues available for cross-linking, Biochem. J. 377 (2004) 407-417.

[32] G.S. Brigidi, S.X. Bamji, Detection of protein palmitoylation in cultured hippocampal neurons by immunoprecipitation and acyl-biotin exchange (ABE), J. Vis. Exp. 72 (2013) e50031, https://doi.org/10.3791/50031.

[33] S.B. Ross-Murphy, Dynamic light scattering. B. J. Berne and R. Pecora, John Wiley, New York, 1976, pp. 376. Price $£ 16.50$, Br. Polym. J. 9 (1977) 177.

[34] M. Blanc, F. David, L. Abrami, D. Migliozzi, F. Armand, J. Burgi, F.G. van der Goot, SwissPalm: protein palmitoylation database, F1000Research 4 (2015) 261.

[35] S. Keerthikumar, D. Chisanga, D. Ariyaratne, H. Al Saffar, S. Anand, K. Zhao M. Samuel, M. Pathan, M. Jois, N. Chilamkurti, L. Gangoda, S. Mathivanan, ExoCarta: a web-based compendium of exosomal cargo, J. Mol. Biol. 428 (2016) 688-692.

[36] S.S. Sanders, D.D. Martin, S.L. Butland, M. Lavallee-Adam, D. Calzolari, C. Kay, J.R. Yates, M.R. Hayden 3rd, Curation of the mammalian palmitoylome indicates a pivotal role for palmitoylation in diseases and disorders of the nervous system and cancers, PLoS Comput. Biol. 11 (2015) e1004405.

[37] M. Ashburner, C.A. Ball, J.A. Blake, D. Botstein, H. Butler, J.M. Cherry, A.P. Davis, K. Dolinski, S.S. Dwight, J.T. Eppig, M.A. Harris, D.P. Hill, L. Issel-Tarver, A. Kasarskis, S. Lewis, J.C. Matese, J.E. Richardson, M. Ringwald, G.M. Rubin, G. Sherlock, Gene ontology: tool for the unification of biology, Gene Ontol. Consort. 25 (2000) 25-29 Nat Genet.

[38] K.H. Cheung, S. Keerthikumar, P. Roncaglia, S.L. Subramanian, M.E. Roth, M. Samuel, S. Anand, L. Gangoda, S. Gould, R. Alexander, D. Galas, M.B. Gerstein, A.F. Hill, R.R. Kitchen, J. Lotvall, T. Patel, D.C. Procaccini, P. Quesenberry, J. Rozowsky, R.L. Raffai, A. Shypitsyna, A.I. Su, C. Thery, K. Vickers, M.H. Wauben, S. Mathivanan, A. Milosavljevic, L.C. Laurent, Extending gene ontology in the context of extracellular RNA and vesicle communication, J. Biomed. Semantics 7
(2016) 19.

[39] C. Gene Ontology, The Gene Ontology (GO) project in 2006, Nucleic Acids Res. 34 (2006) D322-D326.

[40] F.J. Verweij, C. de Heus, S. Kroeze, H. Cai, E. Kieff, S.R. Piersma, C.R. Jimenez, J.M. Middeldorp, D.M. Pegtel, Exosomal sorting of the viral oncoprotein LMP1 is restrained by TRAF2 association at signalling endosomes, J. Extracell. vesicles 4 (2015) 26334.

[41] R. Pires, B. Hartlieb, L. Signor, G. Schoehn, S. Lata, M. Roessle, C. Moriscot, S. Popov, A. Hinz, M. Jamin, V. Boyer, R. Sadoul, E. Forest, D.I. Svergun, H.G. Gottlinger, W. Weissenhorn, A crescent-shaped ALIX dimer targets ESCRT-III CHMP4 filaments, Structure 17 (2009) 843-856.

[42] U.M. Munshi, J. Kim, K. Nagashima, J.H. Hurley, E.O. Freed, An Alix fragment potently inhibits HIV-1 budding: characterization of binding to retroviral YPXL late domains, J. Biol. Chem. 282 (2007) 3847-3855.

[43] W. Ren, U.S. Jhala, K. Du, Proteomic analysis of protein palmitoylation in adipocytes, Adipocytes 2 (2013) 17-28.

[44] J.L. Hernandez, D. Davda, J.D. Majmudar, S.J. Won, A. Prakash, A.I. Choi, B.R. Martin, Correlated S-palmitoylation profiling of Snail-induced epithelial to mesenchymal transition, Mol. BioSyst. 12 (2016) 1799-1808.

[45] R.A. Serwa, F. Abaitua, E. Krause, E.W. Tate, P. O'Hare, Systems analysis of protein fatty acylation in herpes simplex virus-infected cells using chemical proteomics, Chem. Biol. 22 (2015) 1008-1017.

[46] N.M. Chesarino, J.C. Hach, J.L. Chen, B.W. Zaro, M.V. Rajaram, J. Turner, L.S. Schlesinger, M.R. Pratt, H.C. Hang, J.S. Yount, Chemoproteomics reveals Tolllike receptor fatty acylation, BMC Biol. 12 (2014) 91.

[47] S. Li, J. Li, L. Ning, S. Wang, Y. Niu, N. Jin, X. Yao, H. Liu, L. Xi, In silico identification of protein S-palmitoylation sites and their involvement in human inherited disease, J. Chem. Inf. Model. 55 (2015) 2015-2025.

[48] L. Ganesan, I. Levental, Pharmacological inhibition of protein lipidation, J. Membr Biol. 248 (2015) 929-941.

[49] C. Gardiner, D. Di Vizio, S. Sahoo, C. Thery, K.W. Witwer, M. Wauben, A.F. Hill, Techniques used for the isolation and characterization of extracellular vesicles: results of a worldwide survey, J. Extracell. Vesicles 5 (2016) 32945.

[50] C. Thery, S. Amigorena, G. Raposo, A. Clayton, Isolation and characterization of exosomes from cell culture supernatants and biological fluids, Curr. Protoc. Cell Biol. (2006) 22 Chapter 3. Unit 3.

[51] X. Zhou, S. Pan, L. Sun, J. Corvera, S.H. Lin, J. Kuang, The HIV-1 p6/EIAV p9 docking site in Alix is autoinhibited as revealed by a conformation-sensitive antiAlix monoclonal antibody, Biochem. J. 414 (2008) 215-220.

[52] S. Charrin, S. Manie, M. Billard, L. Ashman, D. Gerlier, C. Boucheix, E. Rubinstein, Multiple levels of interactions within the tetraspanin web, Biochem. Biophys. Res. Commun. 304 (2003) 107-112.

[53] S. Sun, L. Sun, X. Zhou, C. Wu, R. Wang, S.H. Lin, J. Kuang, Phosphorylation-dependent activation of the ESCRT function of ALIX in cytokinetic abscission and retroviral budding, Dev. Cell 36 (2016) 331-343.

[54] M.H. Schmidt, I. Dikic, O. Bogler, Src phosphorylation of Alix/AIP1 modulates its interaction with binding partners and antagonizes its activities, J. Biol. Chem. 280 (2005) 3414-3425.

[55] M.R. Dores, H. Lin, J.G. N., F. Mendez, J. Trejo, The alpha-arrestin ARRDC3 mediates ALIX ubiquitination and G protein-coupled receptor lysosomal sorting, Mol. Biol. Cell 26 (2015) 4660-4673.

[56] A. Podesta, G. Tiana, P. Milani, M. Manno, Early events in insulin fibrillization studied by time-lapse atomic force microscopy, Biophys. J. 90 (2006) 589-597.

[57] M. Colombo, C. Moita, G. van Niel, J. Kowal, J. Vigneron, P. Benaroch, N. Manel, L.F. Moita, C. Thery, G. Raposo, Analysis of ESCRT functions in exosome biogenesis, composition and secretion highlights the heterogeneity of extracellular vesicles, J. Cell Sci. 126 (2013) 5553-5565.

[58] A. Llorente, B. van Deurs, K. Sandvig, Cholesterol regulates prostasome release from secretory lysosomes in PC-3 human prostate cancer cells, Eur. J. Cell Biol. 86 (2007) 405-415.

[59] C. Subra, K. Laulagnier, B. Perret, M. Record, Exosome lipidomics unravels lipid sorting at the level of multivesicular bodies, Biochimie 89 (2007) 205-212.

[60] K.W. Witwer, E.I. Buzas, L.T. Bemis, A. Bora, C. Lasser, J. Lotvall, E.N. Nolte-'t Hoen, M.G. Piper, S. Sivaraman, J. Skog, C. Thery, M.H. Wauben, F. Hochberg, Standardization of sample collection, isolation and analysis methods in extracellular vesicle research, J. Extracell. vesicles 2 (2013).

[61] J.A. Bouwstra, G.S. Gooris, W. Bras, H. Talsma, Small angle X-ray scattering: possibilities and limitations in characterization of vesicles, Chemistry and physics of lipids, Vol. 64 1993, pp. 83-98.

[62] M. Hirai, H. Iwase, T. Hayakawa, M. Koizumi, H. Takahashi, Determination of asymmetric structure of ganglioside-DPPC mixed vesicle using SANS, SAXS, and DLS, Biophys. J. 85 (2003) 1600-1610.

[63] S. Castorph, D. Riedel, L. Arleth, M. Sztucki, R. Jahn, M. Holt, T. Salditt, Structure parameters of synaptic vesicles quantified by small-angle $\mathrm{x}$-ray scattering, Biophys. J. 98 (2010) 1200-1208.

[64] M.J.L. Xi Chen, David J. Barlow, Roger J. Morris, Richard K. Heenan, Peter J. Quinn, The structure of detergent-resistant membrane vesicles from rat brain cells, Biochim. Biophys. Acta Biomembr. 1788 (2009) 477-483.

[65] J.D. Nickels, S. Chatterjee, C.B. Stanley, S. Qian, X. Cheng, D.A.A. Myles, R.F. Standaert, J.G. Elkins, J. Katsaras, The in vivo structure of biological membranes and evidence for lipid domains, PLoS Biol. 15 (2017) e2002214.

[66] X. Osteikoetxea, A. Balogh, K. Szabo-Taylor, A. Nemeth, T.G. Szabo, K. Paloczi, B. Sodar, A. Kittel, B. Gyorgy, E. Pallinger, J. Matko, E.I. Buzas, Improved characterization of EV preparations based on protein to lipid ratio and lipid properties, PLoS One 10 (2015) e0121184. 